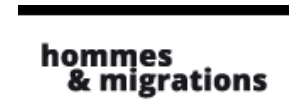

\section{Hommes \& migrations}

Revue française de référence sur les dynamiques

migratoires

$1315 \mid 2016$

Ondes de choc

\title{
Valoriser le capital social des réfugiés par la formation professionnelle
}

Entretien avec Chloé Monin, cheffe de service Emploi et formation au Forum réfugiés-Cosi, et Olivier Marion, directeur du Conservatoire national des arts et métiers en Rhône-Alpes

\section{Marie Poinsot}

\section{(2) OpenEdition \\ Journals}

Édition électronique

URL : http://journals.openedition.org/hommesmigrations/3734

DOI : 10.4000/hommesmigrations.3734

ISSN : 2262-3353

Éditeur

Musée national de l'histoire de l'immigration

Édition imprimée

Date de publication : 1 juillet 2016

Pagination : 117-119

ISBN : 978-2-919040-36-0

ISSN : $1142-852 X$

Référence électronique

Marie Poinsot, «Valoriser le capital social des réfugiés par la formation professionnelle », Hommes \& migrations [En ligne], 1315 | 2016, mis en ligne le 02 janvier 2017, consulté le 15 septembre 2020. URL : http://journals.openedition.org/hommesmigrations/3734 


\title{
VALORISER LE CAPITAL SOCIAL DES RÉFUGIÉS PAR LA FORMATION PROFESSIONNELLE
}

\begin{abstract}
Entretien avec CHLOE MONIN, cheffe de service Emploi et formation au Forum réfugiés-Cosi, et OLIVIER MARION, directeur du Conservatoire national des arts et métiers en Rhône-Alpes, réalisé par MARIE POINSOT, responsable du département des éditions du Musée national de l'histoire de l'immigration et rédactrice en chef de la revue Hommes \& Migrations.
\end{abstract}

Hommes \& Migrations : À quelle occasion le Cnam et Forum réfugiés-Cosi ont-ils souhaité collaborer ensemble dans la région RhôneAlpes ? Quelle était la situation observée sur le terrain ? Quelles activités ces deux organismes ont-ils envisagé de mettre en œuvre pour les réfugiés?

Chloé Monin : Forum réfugiés-Cosi existe depuis 1982. Je m'occupe plus particulièrement du programme Accelair, un programme d'accès à l'emploi et au logement pour l'ensemble des réfugiés du Rhône. Les missions principales de notre association sont l'hébergement et l'accueil des demandeurs d'asile et des réfugiés, la défense du droit d'asile et des droits de l'homme, ainsi qu'une mission d'information et l'aide à l'exercice des droits des personnes retenues dans les centres de rétention administrative. Le premier partenariat avec le Cnam remonte à 2013 sur le volet emploi du programme Accelair. En effet, les bénéficiaires d'une protection internationale rencontrent régulièrement des difficultés pour accéder aux formations proposées dans le cadre du droit commun, car ils ne réunissent pas toujours l'ensemble des prérequis, notamment sur le volet linguistique. Notre premier partenariat avec le Cnam s'est introduit par la mise à disposition gracieuse de salles pour dispenser des cours de français langue étrangère adossés à des cours de théâtre, afin de permettre aux réfugiés d'augmenter leur confiance en eux, d'oser parler pour faciliter leur insertion professionnelle. Une convention de partenariat cadre a été signée entre nos deux organismes. Forum réfugiés-Cosi accompagne 1300 réfugiés, dont certains ont suivi des formations, sont diplômés et ont exercé des métiers dans leur pays d'origine (des ingénieurs, des chefs d'entreprise, etc.), mais dont les compétences ne correspondent pas complètement avec celles exigées sur le marché du travail français. Ils ont donc besoin de suivre certains cours dispensés par le Cnam. Plus de $80 \%$ du public que nous accompagnons étant non francophone, le partenariat avec le Cnam permet d'adosser des modules de français langue étrangère aux formations proposées par le Cnam. Les réfugiés peuvent ainsi acquérir les compétences professionnelles qui leur manquaient pour exercer un métier en France.

H\&M : Quelles sont dans ce partenariat vos interventions réciproques?

C. M. : Le Cnam, Forum réfugiés-Cosi et l'organisme de formation en français qui sera choisi participent à une co-construction du parcours 


\section{INITIATIVES}

de formation des bénéficiaires d'une protection internationale. Sur des métiers très spécialisés, Forum réfugiés-Cosi n'est pas en capacité de savoir si les compétences des personnes correspondent aux besoins des entreprises en France. C'est donc le Cnam qui va mettre en place un pool d'entreprises dans lesquelles les réfugiés pourront faire des stages afin d'identifier les savoirs, les savoir-faire et savoir être de la personne, et ceux qu'elle doit encore acquérir. De nombreuses normes sont nécessaires pour exercer certains métiers : il faut donc passer des habilitations spécifiques, que les réfugiés ne connaissaient pas forcément dans leurs pays d'origine.

H\&M : Comment le Cnam à Lyon s'interroge-til, à travers ce partenariat, sur la manière d'intégrer l'interculturalité dans ses formations professionnelles?

Olivier Marion : Les réfugiés doivent bénéficier d'un accueil digne, professionnel et orienté vers la valorisation de leurs compétences et de leurs savoir-faire pour s'insérer plus facilement dans le monde du travail. Ce n'est pas quand ils arrivent sur le territoire national que ces publics sont prêts à se poser et à réfléchir sereinement à leur projet professionnel. Au préalable, ils ont beaucoup d'urgences à régler avant de se poser cette question d'insertion professionnelle : la santé, y compris mentale, le logement, etc. Face à cette situation, le Cnam n'est pas confronté à son public " classique ", même si nous recevons des demandeurs d'emploi éloignés du travail. C'est une reconstruction citoyenne qu'il nous faut engager reposant sur une démarche d'accueil progressive et respectueuse.

\section{H\&M : L'association Forum réfugiés-Cosi inter- vient-elle aussi dans une démarche d'accompa- gnement social des réfugiés?}

C. M. : Le programme Accelair propose un accompagnement dans le logement à partir d'un dia- gnostic des besoins des réfugiés. L'association a signé un accord collectif avec l'ensemble des bailleurs sociaux du Rhône, avec la métropole de Lyon et avec la préfecture pour que ce public puisse intégrer le parc social. Les travailleurs sociaux vont soutenir les bénéficiaires d'une protection internationale dans leurs démarches administratives, les aider à accéder à leur logement, à tisser des liens avec le territoire. En parallèle, le programme les suit sur le volet emploi avec l'ouverture des droits sociaux, la construction du projet professionnel, l'accompagnement vers des formations professionnelles et/ou linguistique, puis l'accompagnement vers l'emploi.

H\&M : Ces parcours individuels visent-ils toujours le marché du travail ou bien certains mènent-ils à la création d'activités économiques ou d'entreprises ?

C. M. : Le projet professionnel peut effectivement être la création d'une entreprise. Dans certains pays, les réfugiés étaient des petits commerçants, ils ont donc déjà des notions de gestion, d'inventaire, de stocks etc. II faut alors qu'ils accèdent à des formations sur les démarches de création d'entreprise en France. Toutefois, la création d'entreprise est pour l'instant minoritaire dans notre programme, car nous accompagnons les bénéficiaires d'une protection internationale pendant une durée maximale de deux ans après l'obtention de leur statut. Souvent, les personnes vont exercer dans un premier temps un travail en tant que salarié et vont aller vers la création d'entreprise plus tardivement.

H\&M : Avez-vous, parmi votre public, des jeunes ou des femmes qui n'ont aucune qualification professionnelle et qu'il va falloir accompagner de manière plus intense ?

C. M. : Le public du programme Accelair est à $50 \%$ féminin et jeune, puisque plus de la moitié a moins de 35 ans. $37 \%$ ont au moins l'équi- 
valent du baccalauréat. Actuellement, nous accompagnons des personnes de 55 nationalités différentes. Le public est assez hétérogène. Selon les profils, les situations familiales, certains feront le choix d'une insertion professionnelle rapide et nous leur proposerons des formations courtes et techniques (par exemple des FLE métier), d'autres choisiront des parcours de formation plus longs. Enfin, des personnes devront lever de nombreux freins avant une insertion professionnelle.

\section{H\&M : Dans toutes ces démarches, le capital culturel des réfugiés est-il pris en compte par Forum réfugiés-Cosi ? Leur langue d'origine, leur patrimoine culturel, etc. ?}

C. M. : L'objectif du programme est de prendre en compte toutes leurs compétences et pas seulement leurs compétences sociales et professionnelles. Il permet une valorisation de tout le capital personnel acquis également lors de l'exil et qui peut être utile en France, pour travailler et construire une vie sociale.

\section{H\&M : Est-ce que le Cnam s'intéresse à l'accueil que ces publics reçoivent dans les services publics pour leurs premières démarches ?}

0. M. : Le Cnam s'interroge d'abord sur la façon dont nos équipes mettent en place un accueil, une information, une orientation et un accompagnement (AIOA) pour ces publics. Cette première démarche doit permettre de sécuriser le parcours de formation par, notamment, un premier temps de rencontre avec les réfugiés. En cela, nos équipes doivent être formées et préparées à ce type de public pour appréhender leur parcours, leurs expériences professionnelles et, in fine, tenter de les rapprocher des référentiels formation du Cnam en vue d'identifier leurs besoins de formation complémentaire et, peut être dans de rares cas, de déclencher un parcours de réorientation professionnelle vers un autre métier. Ce sont des démarches sur mesure, construites sur une autre temporalité que celle utilisée pour nos publics habituels. À la rentrée, ce dispositif concernera une dizaine de personnes. Pas à pas, nous élaborerons avec eux un projet professionnel adapté, intégrant des périodes d'immersion brève dans une entreprise. À cet égard, nous constituons, en effet, un pool d'entreprises partenaires relevant de plusieurs secteurs (BTP, génie civil, informatique, gestion, etc.), ce qui nous permettra d'identifier, en appui des entreprises, les besoins précis en formation. Ce stage pratique se déroulera en amont de la formation et fera office de bilan actif de compétences. Les premiers retours des entreprises sont positifs ; l'écosystème construit avec Forum réfugiés-Cosi est donc un dispositif formatif en réseau efficace et adapté.

\section{H\&M : Les conditions d'enseignement au Cnam sont-elles favorables aux réfugiés?}

O. M. : Au Cnam, pour pouvoir suivre un cursus de formation, il faut que les réfugiés aient une solide connaissance du français. Cela suppose des adultes motivés. Dans les cours, les auditeurs se soutiennent mutuellement, habités par le même intérêt de progression dans leur projet professionnel et orientés vers un objectif d'insertion sur le marché du travail.

Ils travaillent en binômes ou en trinômes pour réussir leurs études, ils se donnent des informations en réseaux pour trouver un job et ils s'accompagnent mutuellement quand cela est nécessaire. Ce système " organique " se construit dans les cours et finalement bénéficie grandement aux auditeurs, et particulièrement aux réfugiés, contribuant à les faire sortir de leur isolement et du milieu communautaire dans lequel ils ont tendance à se confiner. Cette initiative régionale met en exergue la politique d'engagement de nos deux institutions pour une société plus solidaire envers les personnes les plus vulnérables présentes sur notre territoire. 